\title{
CAPILLARY ELECTROPHORESIS USING COPOLYMERS OF DIFFERENT COMPOSITION AS PHYSICAL COATINGS. A COMPARATIVE STUDY.
}

Guillaume L. Erny ${ }^{\mathrm{a}}$, Carlos Elvira ${ }^{\mathrm{b}}$, Julio San Román ${ }^{\mathrm{b}}$, Alejandro Cifuentes ${ }^{\mathrm{a}^{*}}$

${ }^{\mathrm{a}}$ Institute of Industrial Fermentation (CSIC), Juan de la Cierva 3, 28006 Madrid, Spain

${ }^{\mathrm{b}}$ Institute of Science and Technology of Polymers (CSIC), Juan de la Cierva 3, 28006 Madrid, Spain

* Corresponding author. Tel.: +34-91-562-2900; fax: +34-91-564-4853. E-mail address: acifuentes@ifi.csic.es

Abbreviations: DMA, N,N-dimethylacrylamide; EpyM, ethylpyrrolidine methacrylate; mHBA, m-hydroxybenzoic acid; PFT, partial filling technique; TM, 2,4,6-Trimethylpyridine.

Keywords: Capillary coating, physically adsorbed, protein separation, chiral separation. 


\section{Abstract}

In this work, a comparative study on the use of different polymers as physically adsorbed coatings for Capillary Electrophoresis (CE) is presented. It is demonstrated that the use of ad hoc synthesized polymers as coatings allows tailoring the electroosmotic flow (EOF) in CE increasing the flexibility of this analytical technique. Namely, different polymers were synthesized at our laboratory using different percentages of ethylpyrrolidine methacrylate (EpyM) and N,N-dimethylacrylamide (DMA). Thus, by modifying the percentage of EpyM and DMA monomers it is possible to manipulate the positive charge of the copolymer, varying the global electrical charge on the capillary wall and with that the EOF. These coated capillaries are obtained by simply flushing a given EpyM-DMA aqueous solution into bare silica capillaries. It is shown that by using these coated capillaries at adequate $\mathrm{pHs}$, faster or more resolved $\mathrm{CE}$ separations can be achieved depending on the requirements of each analysis. Moreover, it is demonstrated that these coated capillaries reduce the electrostatic adsorption of basic proteins onto the capillary wall. Furthermore, EpyM-DMA coatings allow the reproducible chiral separation of enantiomers through the partial filling technique. The EpyM-DMA coated capillaries are demonstrated to provide reproducible EOF values independently of the $\mathrm{pH}$ and polymer composition with \% RSD values lower than $2 \%$ for the same day. It is also demonstrated that the coating procedure is reproducible between capillaries. The compatibility of this coating protocol with CE in microchips is discussed. 


\section{Introduction}

The capillary wall has an important contribution to the separation efficiency, resolution and speed in capillary electrophoresis (CE), where fused silica capillaries are generally used. Two different types of phenomena will occur due to the capillary wall. The first one is an induced flow called electroosmotic flow (EOF). The intensity and direction of the EOF will depend on: i) the composition of the capillary wall (or more precisely, on the composition of the capillary wall at the solid-liquid interface), and ii) $\mathrm{pH}$ and composition of the buffer. It is well known that the EOF can modify separation efficiency and separation speed in CE. The EOF can also provide the simultaneous separation of negatively and positively charged analytes. However, in general, the EOF velocity cannot be freely manipulated, since it is usually imposed by the buffer and the negative electrical charge on the capillary wall.

The second type of phenomena, which imposes an additional limitation in CE, is the electrostatic adsorption phenomena having place between analytes with a high positive charge (e.g., basic proteins) and the silica capillary wall with negative charge. Whereas the EOF can be beneficial, analyte-capillary wall adsorption will always reduce the quality of the separation.

Due to these two phenomena, control of the physical properties of the capillary wall is of huge interest in $\mathrm{CE}$, as can be deduced from the numerous works published on this topic [1-17].

In this work, different copolymers synthesized at our laboratory are tested as physical coatings for CE. Namely, the investigated copolymers are composed of ethylpyrrolidine methacrylate$\mathrm{N}, \mathrm{N}$-dimethylacrylamide (EpyM-DMA). One interesting property of these copolymers is that the number of positive charges can be modified as a function of the buffer $\mathrm{pH}$ and the percentage of EpyM. It is demonstrated that CE separations can be tailored by varying these 
parameters, providing higher speed or higher resolution depending on the necessities of each analysis. It is also shown that these coatings can reduce the adsorption phenomena having place between analytes and capillary wall. This is demonstrated using a mixture of basic proteins. Besides, these coatings allow separation of enantiomers (under conditions compatible with e.g., CE-MS) using the partial filling technique. Interestingly, since this coating procedure does not require the use of organic solvents, high viscous solutions or elevated temperatures, its future application to CE separations in microchips seems highly viable.

\section{Experimental Section.}

\subsection{Chemicals.}

Sodium hydroxide, boric acid, formic acid, phosphoric acid, m-hydroxybenzoic acid and 2,4,6trimethylpyridine were from Merck (Darmstad, Germany). Cytochrome C from rabbit, Cytochrome $\mathrm{C}$ from bovine heart, lysozyme from chicken egg-white, 2-[N-morpholine]ethanesulfonic acid (MES) and 3-[cyclohexylamino]-1-propanesulfonic acid (CAPS) were obtained from Sigma (St. Louis, MO, USA). Water was deionized with a Milli-Q system (Millipore, Bedford, MA, USA).

2-Ethyl-(2-pyrrolidine) methacrylate (EpyM) was synthesized by reaction of $\mathrm{N}$-(2hydroxyethyl)-2-pyrrolidine with methacryloyl chloride, both from Fluka (Buchs, Switzerland) and purified by a column chromatography, as previously described [18]. N, NDimethylacrylamide (DMA), purchased from Aldrich (Milwaukee, WI, USA), was vacuum distilled. 2, 2'-Azobisisobutyronitrile (AIBN) from Fluka was purified by fractional 
crystallization from ethanol. Tetrahydrofuran (THF) from Fluka was distilled and dried over molecular sieves. Other reagents were used as received.

\subsection{Polymers synthesis and characterization.}

The monomers EpyM and DMA (see Figure 1) were copolymerized by free radical polymerization at $50^{\circ} \mathrm{C}$, using $\operatorname{AIBN}\left([\mathrm{I}]=1.5 \cdot 10^{-2} \mathrm{~mol} \mathrm{~L}^{-1}\right)$ as a radical initiator and THF ([M]

$=1.0 \mathrm{~mol} \mathrm{~L}^{-1}$ ) as solvent, feed molar fractions of 20, 40, 60, 80 and 100\% EpyM and $24 \mathrm{~h}$ of reaction time. Polymerizations were carried out in a Pyrex ampoule under an oxygen-free $\mathrm{N}_{2}$ atmosphere. Each reaction mixture was precipitated in a large excess of a diethyl ether-hexane mixture, purified by reprecipitation, filtered off and vacuum-dried over phosphorus pentoxide. The copolymers were characterized by ${ }^{1} \mathrm{H}-\mathrm{NMR}$ spectroscopy. Spectra were recorded in $\mathrm{D}_{2} \mathrm{O}$ solutions on a Varian Gemini 200 spectrometer.

\subsection{Buffers preparation.}

Six buffers were designed to determine the EOF mobilities at different $\mathrm{pHs}$, in the different capillaries. Each buffer was made up by mixing $1 \mathrm{M} \mathrm{NaOH}$ with the adequate volume of acid (1 M) in water. The $\mathrm{pH}$ was recorded each time but was uncorrected. This is believed to allow a more reproducible buffer as the $\mathrm{pH}$ is temperature dependant. The buffers used were: $43 \mathrm{mM}$ $\mathrm{NaOH}, 86 \mathrm{mM} \mathrm{H}_{3} \mathrm{PO}_{4}$ at $\mathrm{pH}$ 2.2; $50 \mathrm{mM} \mathrm{NaOH}, 75 \mathrm{mM}$ acid formic at $\mathrm{pH} 3.8 ; 50 \mathrm{mM} \mathrm{NaOH}$, $100 \mathrm{mM}$ MES at pH 6.1; $36 \mathrm{mM} \mathrm{NaOH}, 22 \mathrm{mM} \mathrm{H}_{3} \mathrm{PO}_{4}$ at pH 6.9; $50 \mathrm{mM} \mathrm{NaOH}, 100 \mathrm{mM}$ boric acid at $\mathrm{pH} 8.8 ; 50 \mathrm{mM} \mathrm{NaOH}, 75 \mathrm{mM}$ CAPS at $\mathrm{pH}$ 10.7. All buffers have an ionic strength of $50 \mathrm{mM}$. 
The mixture of proteins was separated in a formate buffer $(25 \mathrm{mM} \mathrm{NaOH}, 50 \mathrm{mM}$ acid formic at $\mathrm{pH}$ 3.6). The FITC derivatized amino acids were separated in an ammonium-acetate buffer (50 $\mathrm{mM}$ ammonium hydroxide to $\mathrm{pH} 6.1$ with $1 \mathrm{M}$ acetic acid) using the partial filling technique. Namely, the buffer inside the capillary contained $1.25 \mathrm{mM}$ vancomycin, while the buffer in the vials was free of vancomycin. This procedure was selected in order to directly transfer the method to CE-MS in the future.

\subsection{Capillary coating.}

The different polymers investigated as coatings were: 100\% EpyM; 80:20 EpyM:DMA; 60:40 EpyM:DMA; 40:60 EpyM:DMA and 20:80 EpyM:DMA. Each polymer was first dissolved in $100 \mathrm{mM}$ formic acid. When fully dissolved, an equivalent volume of $100 \mathrm{mM}$ ammonium hydroxide was added till a final concentration of polymer of $0.1 \mathrm{mg} \mathrm{ml}^{-1}$. In order to obtain a reproducible coating with all the copolymers, the coating procedure was standardized as follows. The new capillary was washed with $0.1 \mathrm{M} \mathrm{NaOH}$ for 20 minutes, then with the polymer solution for 10 minutes, and left to stand inside the capillary for 24 hours. At the beginning of the day the capillary was washed with the polymer solution for 10 minutes. Between the different runs, the capillary was washed with the polymer solution for $2 \mathrm{~min}$, water for 2 min and then the buffer for $3 \mathrm{~min}$.

\subsection{Separation conditions.}

CE experiments were performed using a P/ACE 2050 with UV detection and a P/ACE 2100 with LIF detection, both from Beckman (Fullerton, CA, USA). The capillaries used were bare fused silica with $50 \mu \mathrm{m}$ i.d. and $363 \mu \mathrm{m}$ o.d. (Composite Metals, Worcester, UK.), with $370 \mathrm{~mm}$ of total length (300 $\mathrm{mm}$ detection length). For the chiral amino acids, the capillary was $870 \mathrm{~mm}$ 
of total length (800 mm detection length). Sample injections were performed using a pressure of 0.5 psi. For all experiments the capillary was thermostated at $30^{\circ} \mathrm{C}$.

For the measure of the EOF mobilities, a dissolution of buffer:water:acetone (20:75:5, v:v:v) was used as sample. The EOF mobility was measured for each coating with the six buffers using direct or reverse polarity. For each separation a voltage of $20 \mathrm{kV}$ was applied for 5 min, followed by a pressure separation at $0.5 \mathrm{psi}$ for $8 \mathrm{~min}$. Each run was performed in triplicate. The velocity at 0.5 psi was determined by performing a pressure run for all coatings and with all buffers. Samples were injected for $3 \mathrm{sec}$ and detected at $254 \mathrm{~nm}$. For each coating the same sequence of buffers was used, from the lowest to the highest $\mathrm{pH}$, keeping constant in that way any possible hysteresis effect.

The test mix was composed of tryptophan (Trp), phenylalanine (Phe), tyrosine (Tyr), mhydroxybenzoic acid (m-HBA) and 2,4,6-Trimethylpyridine (TM) all at a concentration of 0.5 $\mathrm{mM}$, dissolved in buffer:water $(1 / 4, \mathrm{v}: \mathrm{v})$. The test mix was analyzed at $20 \mathrm{kV}$. The wavelength was $214 \mathrm{~nm}$, and the sample was injected for $3 \mathrm{~s}$ at $0.5 \mathrm{psi}$.

The proteins sample was made of bovine cytochrome $\mathrm{C}$, rabbit cytochrome $\mathrm{C}$ and lysozyme, all at $0.05 \mathrm{mg} \mathrm{ml}^{-1}$ and dissolved in buffer:water $(1 / 4, \mathrm{v}: \mathrm{v})$. The proteins were separated at $30 \mathrm{kV}$. The sample was injected for $10 \mathrm{~s}$ at $0.5 \mathrm{psi}$, and the detection wavelength was $200 \mathrm{~nm}$.

The chiral amino acids sample was composed of FITC derivatized proline (Pro) and glutamic acids (Glu) in both $\mathrm{L}$ and $\mathrm{D}$ forms dissolved in buffer:water (1:1, v:v) at a concentration of 10 $\mu \mathrm{M}$. Amino acids were derivatized with FITC following the protocol given in reference [19]. The FITC amino acids mix was separated at $25 \mathrm{kV}$. Samples were injected for $5 \mathrm{~s}$ at $0.5 \mathrm{psi}$. The LIF detector was used with $\lambda \cdot$ exc $=488 \mathrm{~nm}$ and $\lambda \cdot{ }_{\text {em }}=520 \mathrm{~nm}$. For the chiral separation a partial 
filling technique was used with vancomycin as the chiral selector. Prior to the separation the capillary was filled with $1.25 \mathrm{mM}$ of vancomycin in ammonium acetate buffer; the separation was carried out using vials filled with buffer without vancomycin.

\section{Results and discussion}

\subsection{Electroosmotic flow using different EpyM-DMA coatings.}

An interesting property of the EpyM-DMA copolymers used in this work (see structure in Figure 1) is that, unlike other positive compounds already used as CE coatings (e.g., polyethyleneimine [12], polybrene [20,21], etc), the number of positive charges on the EpyMDMA copolymer can be adjusted by modifying the ethylpyrrolidine methacrylate content. This property is expected to generate a different behavior of the capillaries depending on the copolymer composition used as coating and, logically, the separation $\mathrm{pH}$. In this way, an additional parameter (i.e., the composition of the copolymeric coating) can be introduced to tailor the separations in CE.

In order to investigate this idea, five different EpyM-DMA copolymers were prepared (namely, 100-0, 80-20, 60-40, 40-60 and 20-80 EpyM-DMA) and once their composition was experimentally corroborated as indicated in Section 2.2, they were used as coatings following the procedure described in Section 2.4. The electroosmotic mobilities provided by these five coated capillaries (plus that from a bare fused silica used as reference) were measured at different $\mathrm{pH}$ values ranging from 2.2 to 10.7 . The results obtained from this experiment are shown in Figure 2. As can be seen, the EOFs obtained for the bare fused silica shows a typical behavior depending on the $\mathrm{pH}$ (i.e., EOF close to zero at very low $\mathrm{pH}$, an increase of $\mathrm{EOF}$ at $\mathrm{pH}$ about 4 and nearly a constant EOF value at $\mathrm{pH}$ values higher than 7). However, the behavior of 
the EpyM-DMA coated capillaries is somewhat different from that found with the bare silica capillary (see Figure 2). Thus, it is observed that all the EpyM-DMA coated capillaries show an anodal EOF at low pHs, a nearly zero EOF at pHs between 6 and 8 and a variable cathodal EOF at higher pHs. This behavior can be explained considering that the global electrical charge of the capillary wall is due to both the amine groups of the EpyM monomer (bearing a positive electrical charge) and the remaining silanol groups onto the silica wall (bearing a negative electrical charge). Thus, under acidic pHs the amine groups are the main charged groups bringing about a global positive charge on the capillary wall and, as a consequence, an anodal EOF. Under very basic pHs the number of positive charges on the polymer decreases and the negative silanol groups become predominant, bringing about a cathodal EOF. It is noteworthy that this EOF is clearly reduced using the EpyM-DMA capillaries (for instance, compare in Figure 2 the EOF values obtained at pH 8 using any coated capillary with that from the bare silica capillary). This is a good indication of the shielding effect of the EpyM-DMA coating even at these basic pHs.

More interestingly, some differences are observed among the coated capillaries depending on the composition of the copolymer used. Thus, from Figure 2, two groups of coated capillaries are clearly distinguishable. On one hand, the group with 20\% and 40\% EpyM that provides a zero EOF at $\mathrm{pH}$ values around 6 . On the other hand, the group of coated capillaries with $60 \%$, $80 \%$ and $100 \%$ EpyM that provides a zero EOF at $\mathrm{pH}$ values around 7.5 . This behavior agrees with the expected higher number of positively charged groups of the latter copolymers. However, this agreement between CE behavior and copolymer composition is not fulfilled at extreme pHs (2.2. and 10.7). For instance, at $\mathrm{pH} 10.7$ the lowest EOF is obtained with the $40 \%$ EpyM coated capillary, when it would be expected that the lowest EOF value should be obtained at that $\mathrm{pH}$ with the $100 \%$ EpyM coated capillary. This result can indicate that some other effects are playing a role (e.g., electrostatic interactions among close charged groups that 
preclude the entrance of new positive charges [22]) resulting in a lower number of positively charged groups than expected.

As a conclusion, Figure 2 demonstrates that the coating composition can indeed be used to tailor the EOF value. For instance, at $\mathrm{pH} 6$ the use of a bare silica capillary, 80\% EpyM coated capillary or $20 \%$ EpyM coated capillary would provide a cathodal, anodal or zero EOF, respectively, without modifying the separation $\mathrm{pH}$. This can be favorably used as an additional parameter to optimize CE separations. This point together with the reproducibility of these types of coatings is explored in the following sections.

\subsection{Reproducibility of the coating.}

In order to study the reproducibility that can be achieved with these coatings, relative standard deviations (\% RSD) were calculated for the analysis time of the EOF obtained after triplicate CE runs carried out at five different $\mathrm{pHs}(2.2,3.8,6.9,8.8$ and 10.7). Figure 3 shows the RSD values obtained depending on the $\mathrm{pH}$ and type of capillary used (five coated capillaries plus a bare silica tube). Although the RSD values were better using the bare fused silica capillary at pHs above 6.9, it can be observed that the use of EpyM-DMA coated capillaries clearly improved the RSD values at $\mathrm{pH} 2.2$ and 3.8. Moreover, as can also be seen in Figure 3, the RSD values using coated capillaries were lower than $2 \%$ in all cases. This effect seems to be related to the magnitude of the EOF independently of its direction towards the anode or the cathode (i.e., the higher the EOF value the better the time reproducibility that can be achieved in CE).

In order to corroborate the reproducibility of the coating protocol, six bare capillaries were coated using the protocol described in Section 2.4. Namely, three capillaries were coated using a 20-80 EpyM-DMA copolymer and the other three with an 80-20 EpyM-DMA copolymer. The 
results are shown in Figure 4A and B, respectively. Although some slight variation can be observed among capillaries from the same group, the same behavior described in Figure 2 was obtained (see for instance the different EOFs obtained for the two types of capillaries at $\mathrm{pH}$ around 6), corroborating the reproducibility of the coating protocol.

To study the stability of the coating and the effect of the recoating between runs, multiple injections were carried out using m-HBA as analyte in the phosphate buffer at $\mathrm{pH} 6.9$. The $20 \%$ EPyM coated capillary was used for this study. For the first five injections, the washing procedure described under experimental was used (i.e. polymer solution for $2 \mathrm{~min}$, then water for $2 \mathrm{~min}$, and BGE for $3 \mathrm{~min}$ ). The following 15 injections were then performed without washing the capillary with the polymer solution. Good RSDs $(n=5)$ for both the migration time $(0.37 \%)$ and corrected peak area $(0.98 \%)$ were obtained when the full washing procedure was used. However, when the wash step with the polymer solution was omitted, a slight shift of the migration time was observed. This intensity of the shift was small, and relatively constant $(0.5 \pm$ $0.2 \mathrm{~s}$ per injection), leading to a variation of $5 \%$ in the migration time (from 2.24 to $2.41 \mathrm{~min}$ ) after 15 injections. As the removal of the coating will be dependant on the BGE used, the same experiments were performed using the borate buffer at $\mathrm{pH}$ 8.8. As previously shown, good RSDs $(n=5)$ were obtained for both the migration time $(0.62 \%)$ and corrected peak area $(0.63 \%)$ when the full washing procedure was used. As expected, the shift was higher $(2.7 \pm 1.8$ s per injection) than with the phosphate buffer, leading in this case to a variation of $18 \%$ in the migration time (from 2.86 to $3.49 \mathrm{~min}$ ) for the 15 injections. Both series of experiments show that the coating needs to be regenerated between injections in order to achieve reproducible results. 


\subsection{Applications of EpyM-DMA coated capillaries.}

In order to demonstrate the possibilities of the use of EpyM-DMA copolymers as coatings in $\mathrm{CE}$, the following applications were carried out. Thus, Figure 5 shows a comparison of the CE separations achieved under identical conditions using a bare fused silica capillary and a $60 \%$ EpyM coated capillary at $\mathrm{pH}$ 6.9. Although the high EOF mobility obtained with the bare silica capillary allows simultaneous separation of positively and negatively charged analytes (TM is positively charged and the other analytes are negatively charged at this $\mathrm{pH}$ ), a low resolution is also observed. Thus, Trp (peak 1), Phe (peak 2) and Tyr (peak 3) were not resolved under these conditions. By using the $60 \%$ EpyM coated capillary it was possible to slow down the EOF, improving the resolution and allowing the separation of Trp, Phe and Tyr at the same separation $\mathrm{pH}$ (see Figure 5). This could have interesting applications, such as the high resolved separation of analytes that are only stable at physiological $\mathrm{pH}$ values.

Since all the components analyzed in Figure 5 were stable in a broader $\mathrm{pH}$ range, in this case the EOF in the bare fused silica capillary could be slowed down by working at a lower $\mathrm{pH}$ and thus the resolution improved. As shown in Figure 6, Trp, Phe and Tyr were baseline separated in a bare fused silica capillary at $\mathrm{pH} 2.2$. In this case, the use of the $60 \%$ EpyM capillary could also offer an interesting advantage since it provides much faster separations, making possible the baseline separation of these three compounds in about 4 minutes. In this separation, as well as in the run at $\mathrm{pH} 6.9$ discussed above, the polarity was reversed when using the $60 \%$ EpyM capillary. This can explain the different order of migration obtained with the bare silica capillary and the $60 \%$ EpyM capillary.

An additional advantage of these EpyM-DMA coated capillaries is the possibility to use them to carry out separations of basic proteins in CE. One of the main problems during the separation of 
basic proteins by $\mathrm{CE}$ is the electrostatic interaction between the positively charged proteins and the negatively charged wall. Basic proteins can be separated at very low $\mathrm{pH}$ decreasing the degree of ionization of the silica surface. Alternatively, the capillary can be coated to obtain a neutral or a positively charged surface allowing basic proteins separation. The use of the EpyMDMA coating provides a positively charged wall at pHs between 2 and 7.5 and whose EOF mobility can be tailored to optimize resolution and separation speed by choosing the adequate polymer composition and buffer $\mathrm{pH}$. This is demonstrated in Figure 7, where three basic proteins (lysozyme, cytochrome $\mathrm{C}$ from rabbit and bovine cytochrome $\mathrm{C}$ ) are separated in a sodium formate buffer at $\mathrm{pH} 3.6$, using both a bare silica capillary and a $60 \%$ EpyM coated capillary. As can be observed in Figure 7, neither separation nor signal were observed using the bare silica capillary due to the irreversible adsorption of these proteins onto the capillary wall. At the same $\mathrm{pH}$, the $60 \%$ EpyM capillary provides a high electroosmotic mobility (Figure 2). Therefore, this capillary should provide at that $\mathrm{pH}$ a fast separation, but also a highly positive charged surface, which should protect efficiently against proteins adsorption. This is demonstrated in Figure 7, where the same 3 proteins at low concentration $(50 \mu \mathrm{g} / \mathrm{ml})$ could be well separated in less than 5 minutes showing a symmetrical peak shape when the EpyM-DMA coated capillary was used.

EpyM-DMA coatings can also be utilized in CE to facilitate the use of the so-called partial filling technique (PFT). This technique is used to avoid that a compound that is present in the capillary but not in the running buffer vials can contaminate the detector. The PFT approach is now principally used together with CE-MS to avoid that non-volatile compounds, mainly chiral selectors and surfactants, contaminate the ionization source [23-24]. An important point to consider is that a suitable and reproducible suppression of the EOF is still a challenging issue in CE when using chiral selectors and PFT [7]. According to Figure 2, capillaries coated with 20 or 40\% EpyM should provide zero EOFs at $\mathrm{pH}$ values around 6. This property could be favorably 
used to develop a PFT suitable, for e.g., to separate chiral compounds that at the mentioned $\mathrm{pH}$ bear an electrical charge opposite to that of the chiral selector. This is the case of L/D amino acids derivatized with FITC (negatively charged at $\mathrm{pH}$ 6) and vancomycin, a chiral selector positively charged at the mentioned $\mathrm{pH}$ [25-26]. Moreover, in order to obtain an adequate $\mathrm{CE}$ separation of enantiomers, interactions between the solutes and the chiral selector must be enhanced [27]. These points are demonstrated in Figure 8. Thus, Figure 8A shows, as expected, that no chiral separation could be obtained using a coated capillary and no vancomycin into the running electrolyte. Figure $8 \mathrm{~B}$ shows that the use of a bare fused silica capillary and vancomycin added to the CE electrolyte does not provide a chiral separation either, since solutes and chiral selectors are pushed by the cathodal EOF preventing their adequate interaction. The chiral separation can be achieved using a 20\% EpyM coated capillary together with vancomycin (see Figure 8C). Under these conditions, the coating suppresses the EOF, increasing in that way the analyte-chiral selector interactions bringing about their separation. Moreover, it is wellknown that vancomycin can electrostatically adsorb onto fused silica giving rise to irreproducible results [28]. This limitation is also overcome by using EpyM-DMA coated capillaries, obtaining RSD values lower than $0.05 \%(n=3)$ for the separation of FITC-L/D-Glu and FITC-L/D-Pro.

\section{Concluding remarks}

A simple and reproducible procedure to coat capillaries has been developed. The coating is physically adsorbed onto the capillary wall and it is regenerated just by flushing the capillary with a dilute solution containing the polymer between injections. It has been demonstrated that the adequate choice of the EpyM-DMA composition used as coating gives an additional parameter for tailoring the separations in CE. It has been shown that the adequate selection of 
the coating can: i) improve analysis time reproducibility, ii) enhance resolution, iii) increase analysis speed, iv) diminish the negative adsorption between solutes and capillary wall and, v) make feasible the application of partial filling techniques (e.g., to separate chiral solutes). This coating does not require the use of organic solvents, high viscous solutions or elevated temperatures; therefore, its future application to CE separations of proteins in microchips can be anticipated. 


\section{References}

[1] Shou, C. Q., Zhou, C. L., Zhao, C. B., Zhang, Z. L., Li, G. B., Chen, L. R., Talanta 2004, $63,887-891$.

[2] González, N., Elvira, C., San Román, J., Cifuentes, A., J. Chromatogr. A 2003, 1012, 95101.

[3] Strelec, I., Pacáková, V., Bosáková, Z., Coufal, P., Guryca, V., Stulik, K., Electrophoresis 2002, 23, 528-535.

[4] López-Soto-Yarritu, P., Diez-Masa, J. C., Defrutos, M., Cifuentes, A., J. Sep. Sci. 2002, 25, 1112-1118.

[5] Righetti, P. G., Gelfi, C., Verzola, B., Castelletti, L., Electrophoresis 2001, 22, 603-611.

[6] Horvath, J., Dolník, V. Electrophoresis 2001, 22, 644-655.

[7] Chiari, M., Cretich, M., Desperati, V., Marinzi, C., Galbusera, C., De Lorenzi, E., Electrophoresis 2000, 21, 2343-2351

[8] Katayama, H., Ishihama, Y., Asakawa, N., Anal. chem. 1998, 70, 5272-5277.

[9] Cifuentes, A., Diez-Masa, J. C., Fritz, J., Anselmetti, D., Bruno, A. E., Anal. chem. 1998, 70, 3458-3462.

[10] Cifuentes, A., Poppe, H., Kraak, J. C., Erim, F. B., J. Chromatogr. B 1996, 21-27

[11] Cifuentes, A., Rodriguez, M. A., Garciamontelongo, F. J., J. Chromatogr. A 1996, 742, 257-266.

[12] Erim, F. B., Cifuentes, A., Poppe, H., Kraak, J. C., J. Chromatogr. A 1995, 708, 356-361.

[13] Cifuentes, A., Santos, J. M., Defrutos, M., Diez-Masa, J. C., J. Chromatogr. A 1993, 652, 161-170.

[14] Towns, J. K., Regnier, F. E., Anal. chem. 1991, 63, 1126-1132.

[15] Towns, J. K., Regnier, F. E., J. Chromatogr. 1990, 516, 69-78. 
[16] Hjerten, S., Electrophoresis 1990, 11, 665-690.

[17] Bruin, G. J. M., Chang, J. P., Kuhlman, R. H., Zegers, K., Kraak, J. C., Poppe, H., J. Chromatogr. 1989, 471, 429-436.

[18] González, N., Elvira, C., San Román, J., J. Polym. Sci. Part A: Polym. Chem. 2003, 41, $395-407$.

[19] Simó, C., Barbas, C., Cifuentes, A., J. Agric. Food. Chem. 2002, 50, 5288-5293.

[20] Mizzen, C. A., Mclachlan, D. R., Electrophoresis 2000, 21, 2359-2367.

[21] Liu, X., Erickson, D., Li, D., Krull, U. J., Anal. Chim. Acta 2004, 507, 55-62.

[22] Cifuentes, A., Poppe, H., J. Chromatogr. A 1994, 680, 321-340.

[23] Shamsi, S. A., Miller, B. E., Electrophoresis 2004, 25, 3927-3961.

[24] Shamsi, S. A., Electrophoresis 2002, 23, 4036-4051.

[25] Fanali, S., Desiderio, C., Schulte, G., Heitmeier, S., Strickmann, D., Chankvetadze, B., Blaschke, G., J. Chromatogr. A 1998, 800, 69-76.

[26] Tanaka, Y., Kishimoto, Y., Terabe, S., J. Chromatogr. A 1998, 802, 83-88.

[27] Chankvetadze, B., Capillary Electrophoresis in Chiral Analysis, John Wiley \& Sons, Chinchester, 1997.

[28] Desiderio, C., Fanali, S., J. Chromatogr. A 1998, 807, 37-56 


\section{FIGURES}

Figure 1. Structure of the ethylpyrrolidine methacrylate-N,N-dimethylacrylamide (EpyMDMA) copolymer.

Figure 2. Electroosmotic mobility as a function of $\mathrm{pH}$ and type of capillary used. See Experimental for details.

Figure 3. Relative standard deviation $\left(\% \mathrm{RSD}_{\mathrm{n}=3}\right)$ of the electroosmotic mobility determination as a function of the $\mathrm{pH}$ and type of capillary used. See Experimental for details.

Figure 4. Electroosmotic mobility reproducibility obtained with A) three 20-80 EpyM-DMA coated capillaries and B) three 80-20 EpyM-DMA coated capillaries. Rest of conditions as in Figure 2.

Figure 5. Comparison of the separation of 1) Trp, 2) Phe, 3) Tyr, 4) TM and, 5) m-HBA using a bare silica capillary and a 60-40 EpyM-DMA coated capillary. Running buffer: $36 \mathrm{mM} \mathrm{NaOH}$, $22 \mathrm{mM} \mathrm{H}_{3} \mathrm{PO}_{4}$ at $\mathrm{pH}$ 6.9. The sample was injected for $3 \mathrm{~s}$ at 0.5 psi. The separation was performed in normal polarity with the bare silica capillary $(20 \mathrm{kV})$ and reverse polarity with the coated capillary $(-20 \mathrm{kV})$. See Experimental for rest of conditions

Figure 6. Comparison of the separation of 1) Trp, 2) Phe and, 3) Tyr using a bare silica capillary and a 60-40 EpyM-DMA coated capillary. Running buffer: $43 \mathrm{mM} \mathrm{NaOH}, 86 \mathrm{mM} \mathrm{H}_{3} \mathrm{PO}_{4}$ at $\mathrm{pH}$ 2.2; sample injected for $3 \mathrm{sec}$ at $0.5 \mathrm{psi}$. The separation was performed in normal polarity with the bare silica capillary $(20 \mathrm{kV})$ and reverse polarity with the coated capillary $(-20 \mathrm{kV})$. 
Figure 7. Separation of basic proteins using a bare silica capillary and a 60-40 EpyM-DMA coated capillary. Running buffer: $25 \mathrm{mM} \mathrm{NaOH}, 50 \mathrm{mM}$ acid formic at $\mathrm{pH}$ 3.6. Running voltage: $30 \mathrm{kV}$. Injection of 1) Lysozyme, 2) Rabbit cytochrome C, and 3) Bovine cytochrome C for $10 \mathrm{~s}$ at $0.5 \mathrm{psi}$ all of them at $0.05 \mathrm{mg} \mathrm{ml}^{-1}$. See Experimental for rest of conditions.

Figure 8. Chiral separation of FITC-derivatized amino acids using A) a 20-80 EpyM-DMA coated capillary and no vancomycin in the running buffer; B) bare fused silica capillary and 1.25 $\mathrm{mM}$ vancomycin in the running buffer and; C). 20-80 EpyM-DMA coated capillary and 1.25 $\mathrm{mM}$ vancomycin in the running buffer. Running buffer: $50 \mathrm{mM}$ ammonium acetate buffer at $\mathrm{pH}$ 6.1. Running voltage: $25 \mathrm{kV}$ (B: direct polarity, $\mathrm{A}$ and $\mathrm{C}$ : reverse polarity). The sample, composed of $10 \mu \mathrm{M}$ FITC-D/L-Glu and $10 \mu \mathrm{M}$ FITC-D/L-Pro, was injected at 0.5 psi for $5 \mathrm{~s}$. LIF detection was used with $\lambda_{\text {.exc }}=488 \mathrm{~nm}$ and $\lambda_{\cdot \mathrm{em}}=520 \mathrm{~nm}$. See Experimental for rest of conditions. 


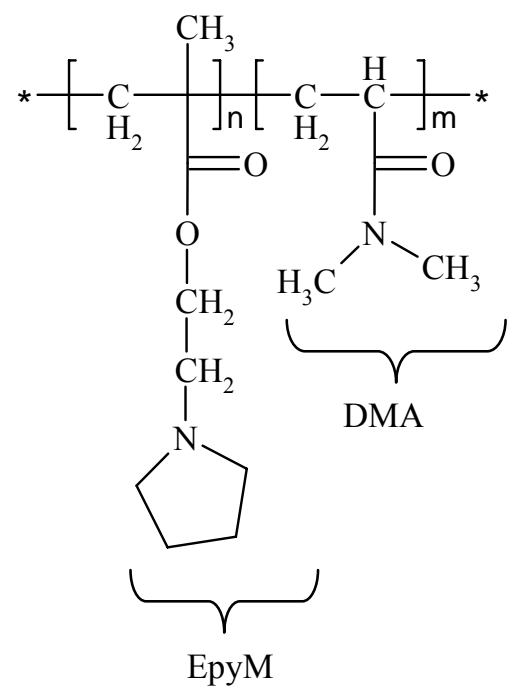

Figure 1. 


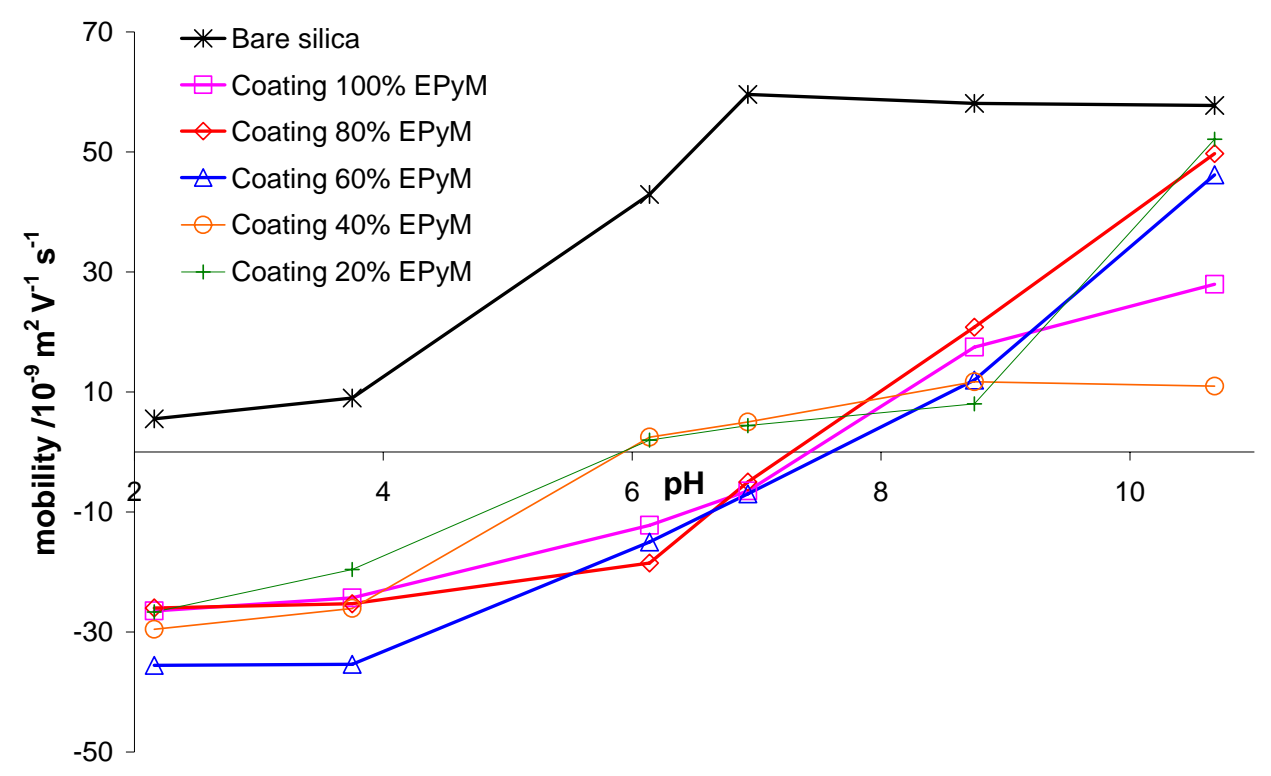

Figure 2. 


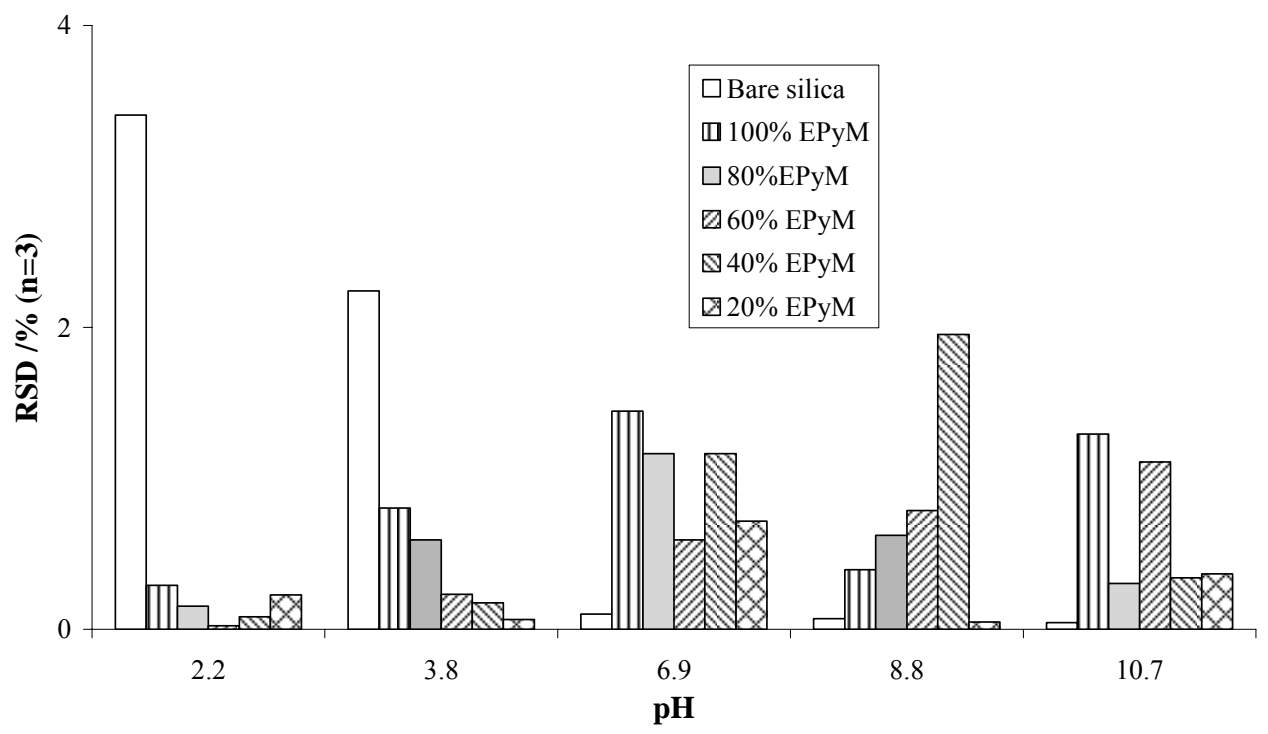

Figure 3. 

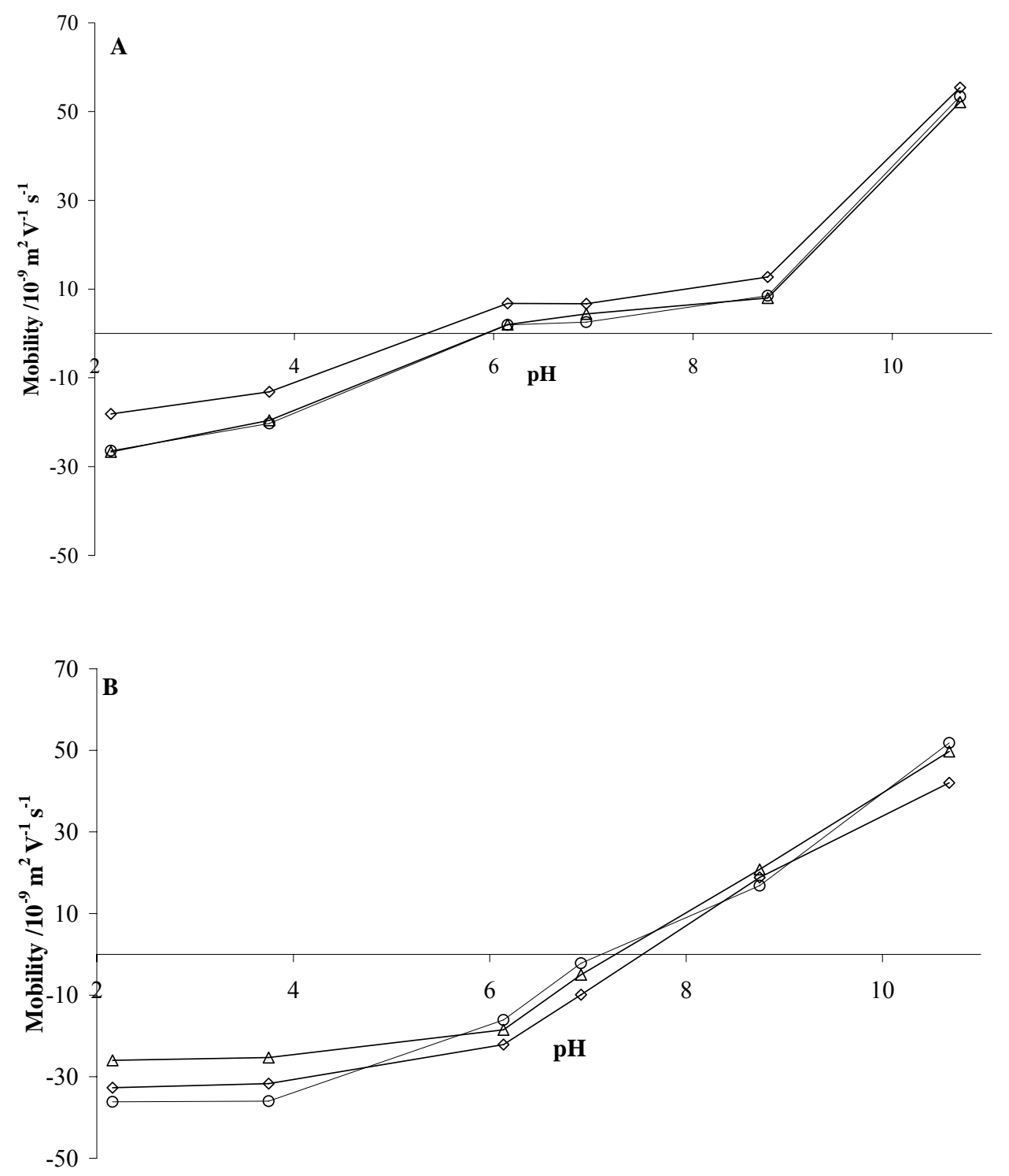

Figure 4. 


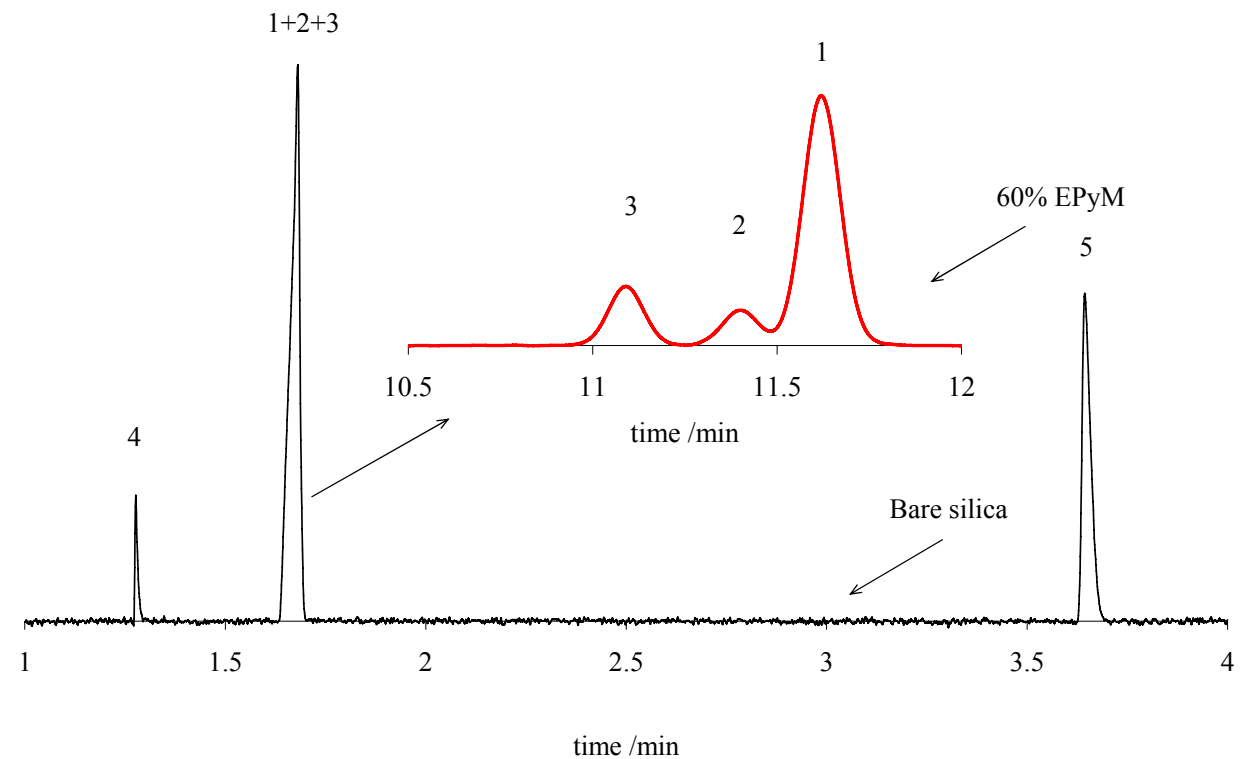

Figure 5. 


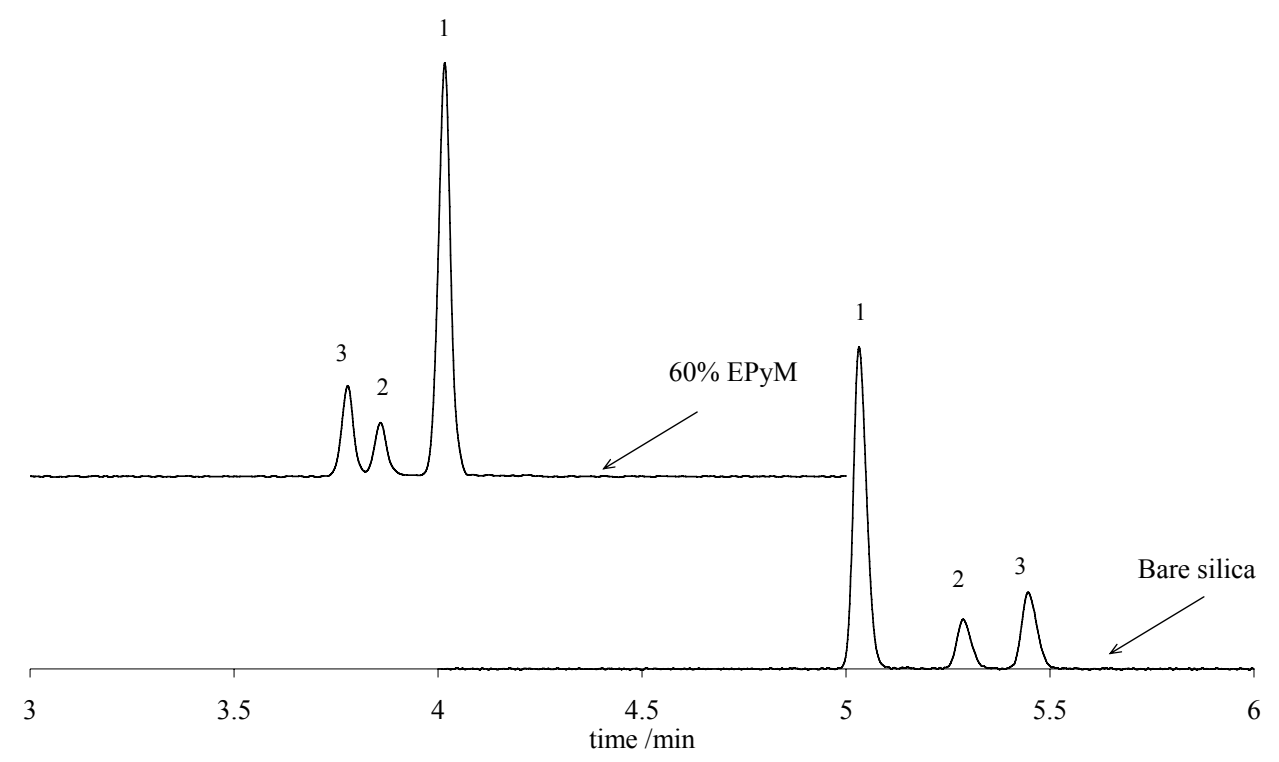

Figure 6. 


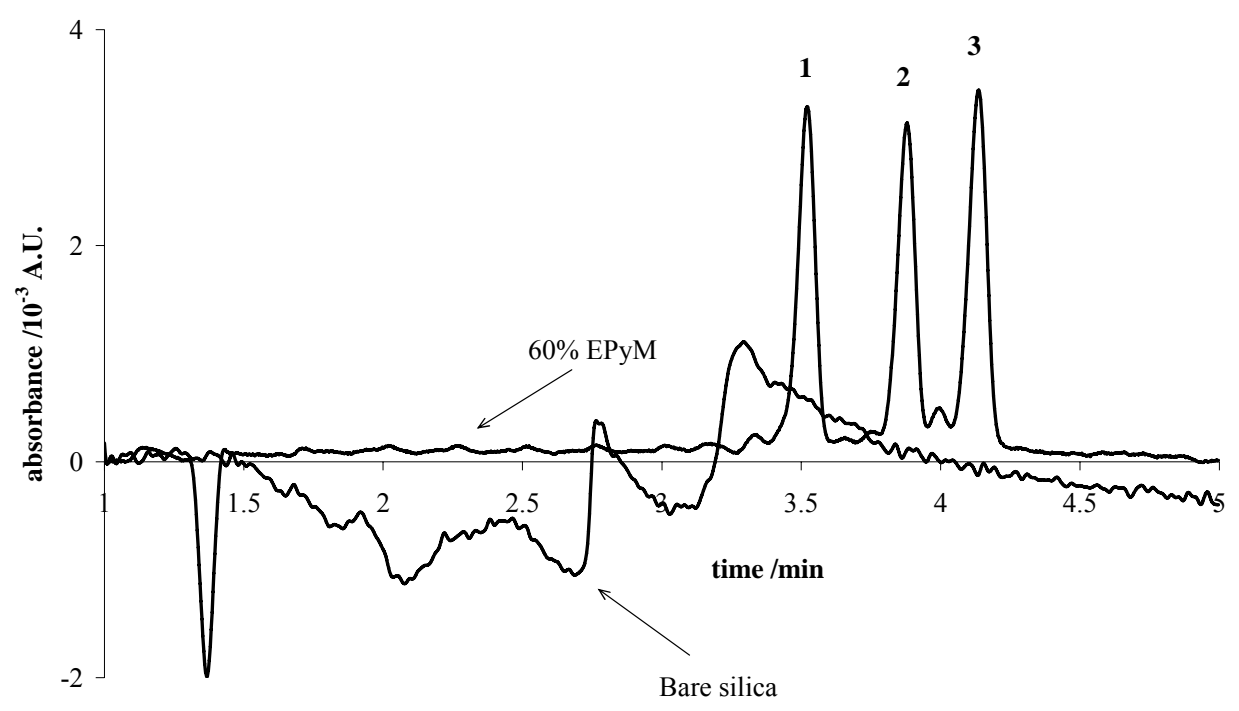

Figure 7. 

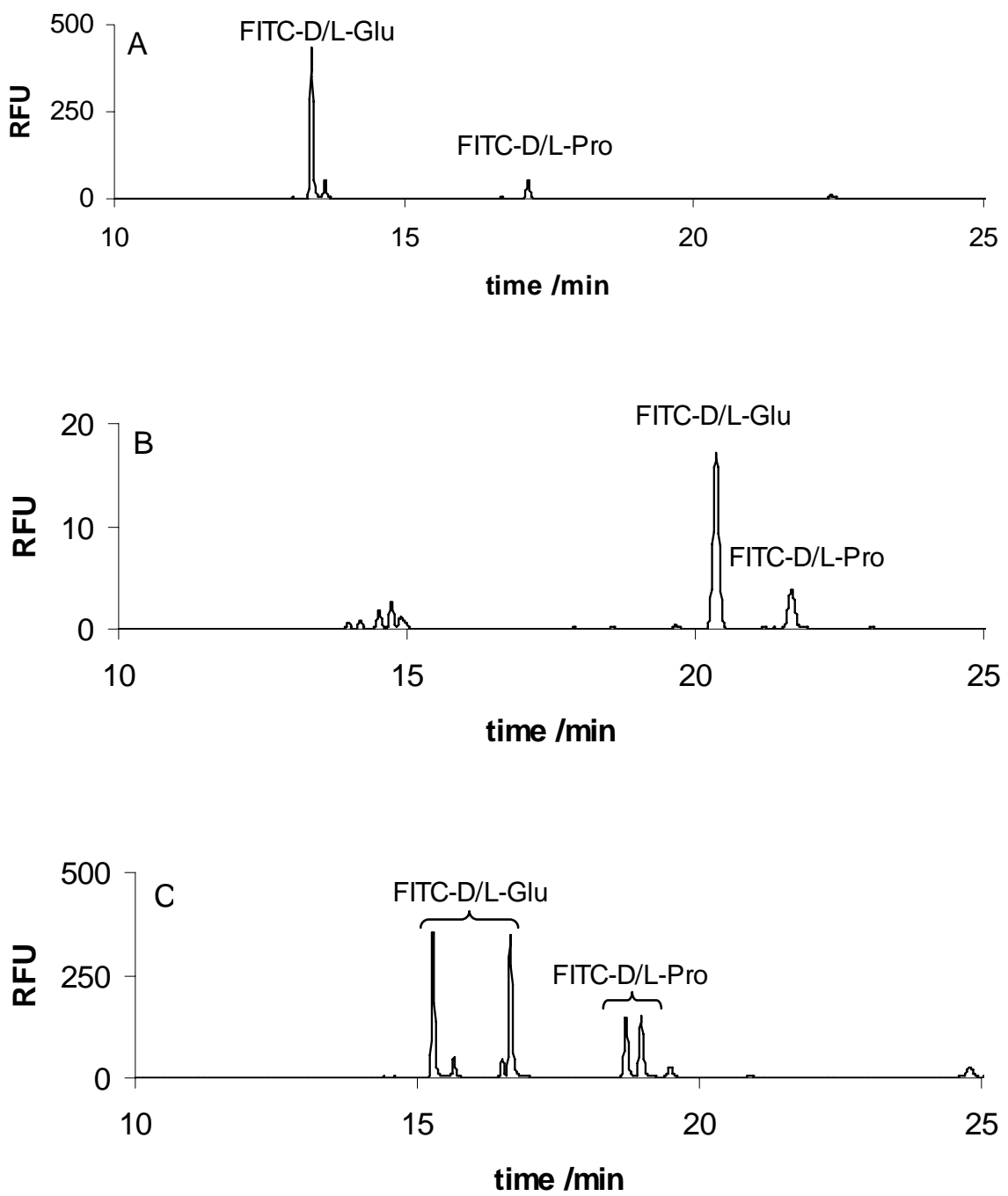

Figure 8. 